\title{
Synthesis of Carbon Nanotube Incorporated Molecular Imprinted Polymer with Binding Affinity towards Testosterone
}

\author{
Anju Augustine and Beena Mathew \\ School of Chemical Sciences, Mahatma Gandhi University, Kottayam, Kerala 686560, India \\ Correspondence should be addressed to Beena Mathew; beenamscs@gmail.com
}

Received 19 November 2013; Accepted 9 January 2014; Published 19 February 2014

Academic Editors: C. Chen, Y. Chen, and B. G. Soares

Copyright (C) 2014 A. Augustine and B. Mathew. This is an open access article distributed under the Creative Commons Attribution License, which permits unrestricted use, distribution, and reproduction in any medium, provided the original work is properly cited.

\begin{abstract}
A novel polymer was synthesised using functionalized carbon nanotube and acrylamide as the polymer support for the separation of testosterone. The developed polymers were characterised using FT-IR, XRD, and SEM techniques. Imprinted polymer showed specificity towards the template testosterone. Among the various polymers, the MWCNT incorporated polymer showed high binding towards the used template. Investigation of the selectivity characteristics revealed that the developed polymer showed selectivity toward the template testosterone than similar compounds. The bound template could be totally recovered and regenerated polymer maintains its recognition property after repeated use. On the basis of the results, the imprinted polymer can be applied for the direct extraction of testosterone in clinical analysis.
\end{abstract}

\section{Introduction}

Molecular imprinting has become an effective way to prepare polymer materials that show a "memory effect" toward their templates [1]. Compared to other recognition systems, MIPs possess many promising characteristics, such as low cost and easy synthesis, high stability to harsh chemical and physical conditions, and excellent reusability [2]. The molecular imprinting technique, first proposed by Wulff and Sarhan in 1972 [3], is one of the promising and facile methods to impart molecular recognition sites in synthetic polymers. Molecular imprinted polymers (MIPs) have been used for molecular recognition ranging from small molecules $[4,5]$ to macromolecules $[6,7]$. The improper or illegal use of hormones as veterinary drugs may result in unwanted residues in food products derived from livestock breeding. However the direct detection of target corticosteroids in complex biological matrices can be a difficult task, and sample cleanup treatments are frequently necessary before performing the instrumental analysis. To extract synthetic corticosteroids from biological samples using the MIP approach efficiently, an imprinted polymer should be able to selectively recognise the main analytical target by making use of several noncovalent interactions. Multiwalled carbon nanotubes (MWNTs), which were first discovered in 1991 [8], are widely considered the quintessential nanomaterial. Due to their high strength, extremely large surface areas, and unique chemical properties, MWNTs can serve as a reinforcing element or the core when fabricating core-shell structural MIPs. A thin layer of MIPs can be polymerized onto the surfaces of MWNTs. Thus, binding cavities in the thin MIPs outer layer can greatly improve the accessibility to template molecules.

Testosterone (TSN) is a naturally occurring estronic compound and a product of cholesterol by a long biosynthetic pathway. It is produced mainly in ovaries, also in adrenal cortex, brain, adn testes and during pregnancy in placenta. In humans TSN is produced naturally in both sexes, but women have much higher concentration [9]. Testosterone is required for the normal functioning and native circulation in the human body, but carcinogenic effects are possible at enhanced levels [10]. Testosterone presents several binding sites capable of interacting via $\mathrm{H}$-bond with the amide and carboxyl groups of the synthesised polymers. At proper level TSN is necessary for normal functioning and at elevated levels they can be toxic and carcinogenic because they are growth promoters [11]. The analysis of testosterone in urine specimens can support the diagnosis of diseases based on irregularities in the steroid profile. Due to the ease of 


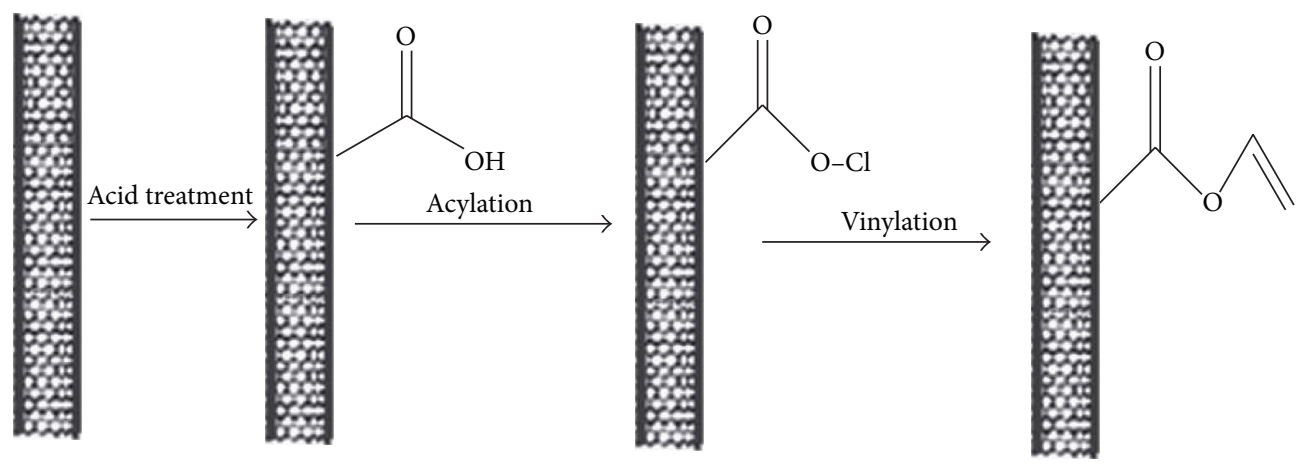

SCHEME 1: Functionalization of MWCNT.

collection and the voluminous presence of metabolites, urine specimens constitute ideal material for human studies. These compounds are present in urine at very low concentration levels $\left(\mathrm{ngmL}^{-1}\right)[10]$.

The present paper deals with the investigation of the binding capacity and the influence of carbon nanotube on the binding of testosterone. Also discussed is the detection method of TSN in urine, if it is present in abnormal level. The carbon nanotube was functionalized using various steps. The TSN imprinted and nonimprinted polymers were investigated for their specific and selective binding of TSN.

\section{Experimental}

2.1. Materials and Methods. MWCNTs (internal diameter 2-6 nm, outer diameter $10-15 \mathrm{~nm}$, length $0.1-10 \mathrm{~m}$, and purity $>90 \%$ ), ethylene dimethacrylate (EGDMA), and 2,2 azoisobutyronitrile (AIBN) were purchased from SigmaAldrich-Germany). Thionyl chloride $\left(\mathrm{SOCl}_{2}\right)$, dimethlysulfoxide (DMSO), dimethylformamide (DMF), tetrahydrofuran (THF), and triethylamine (TEA) were obtained from Merck, Germany. Triethylamine (TEA), acrylamide (AM), and acetic acid (HOAc) were obtained from SRL (India). Progesterone, testosterone, and cholesterol were purchased from Sigma-Aldrich (Germany) and used as received.

The polymer morphology was investigated by scanning electron microscopy (SEM) using a JEOL-JSM-6390 A microscope. Binding studies were carried out using Shimadzu UVvis. Spectrophotometer. The FT-IR studies were carried out using Perkin-Elmer spectrum 400 FT-IR spectrophotometer. Crystalline nature was studied using X-ray diffractogram which was recorded by PAN analytic XPERT PRO.

2.1.1. Modification of MWCNTs. The modification of MWCNTs is based on the protocols suggested in Scheme 1.

2.1.2. Acid Treatment of MWCNTs. MWCNTs ( $0.5 \mathrm{~g})$ were oxidized with $60 \mathrm{~mL}$ of concentrated nitric acid solution at $100^{\circ} \mathrm{C}$ for $12 \mathrm{~h}$. After cooling to room temperature, the mixture was filtered and washed thoroughly with distilled water several times until the $\mathrm{pH}$ of the final wash (filtrate) came down to neutral. The filtered solid was dried under vacuum to obtain carboxylic acid functionalized MWCNTs
(MWCNTs-COOH) [FT-IR $\left(\mathrm{cm}^{-1}\right): 3586(-\mathrm{COOH}), 2921(-$ $\mathrm{CH}), 1713$ (-C O), 1129 (-C-OH)].

2.1.3. Acylation of $M W C N T s-C O O H$. Functionalized MWCNTs $(0.4 \mathrm{~g})$ were suspended in the mixture of $10 \mathrm{~mL} \mathrm{SOCl}$ and $30 \mathrm{~mL}$ chloroform for $24 \mathrm{~h}$ reflux condition at $60^{\circ} \mathrm{C}$. Excess $\mathrm{SOCl}_{2}$ was removed by multiple washings of the solid with THF and then dried under vacuum to obtain MWCNTsCOCl [FT-IR $\left(\mathrm{cm}^{-1}\right)$ : 2921 (-CH), 1794 (-C O), 855 (-C-Cl)].

2.1.4. Vinylation of $\mathrm{MWCNTs-COCl}$. MWCNTs-COCl $(0.2 \mathrm{~g})$ was dispersed in $30 \mathrm{~mL}$ THF and then $20 \mathrm{~mL}$ allylamine, dissolved in $10 \mathrm{~mL}$ of DMF, was added dropwise to the mixture. The mixture was stirred at $60^{\circ} \mathrm{C}$ for $24 \mathrm{~h}$ and the solid collected after centrifugation was washed with THF. Finally the resulting solid was vacuum-dried to obtain vinyl group functionalized MWCNTs (MWCNTs-CH-CH $)$ [FT-IR $\left(\mathrm{cm}^{-1}\right): 3300,2993(-\mathrm{NH}), 2866(-\mathrm{CH}), 1680$ (amide I), 1585 (-C-C-), 1561 (amide II), 1254 (-C-N-)].

2.1.5. Synthesis of MWCNTs-MIP Template Adduct. Development of MIP onto the vinyl group functionalized MWCNTs surface is depicted in Scheme 2. Accordingly, the monomer (AM, $0.4 \mathrm{mmol} / 2 \mathrm{~mL} \mathrm{DMSO}$ ), template (TSN, $0.1 \mathrm{mmol} / 2 \mathrm{~mL} \mathrm{DMSO}$ ), and the cross-linker (EGDMA, $2 \mathrm{mmol}$ ) were mixed together in a test tube followed by the addition of $60 \mathrm{mg}$ MWCNTs- $\mathrm{CH}=\mathrm{CH}_{2}$ (dispersed in DMSO). To this prepolymerization mixture, AIBN $(0.1 \mathrm{mmol})$ was added and the mixture was purged with $\mathrm{N}_{2}$ gas for $10 \mathrm{~min}$. Then the test tube was sealed and cured at $37^{\circ} \mathrm{C}$ for $10 \mathrm{~h}$. Nonimprinted polymer (NIP) was prepared following the above procedure but in the absence of template. In order to reveal the specialty of MWCNTs, an imprinted and nonimprinted polymer was also prepared without carbon nanotube by following the same procedure as described above.

2.1.6. Binding Studies. In order to evaluate the recognition properties of the polymer towards the target molecule, binding studies were carried out by batch experiments. Testosterone sample solution $(7 \mathrm{~mL})$ was allowed to contact with the imprinted and control polymer of same dimension. 


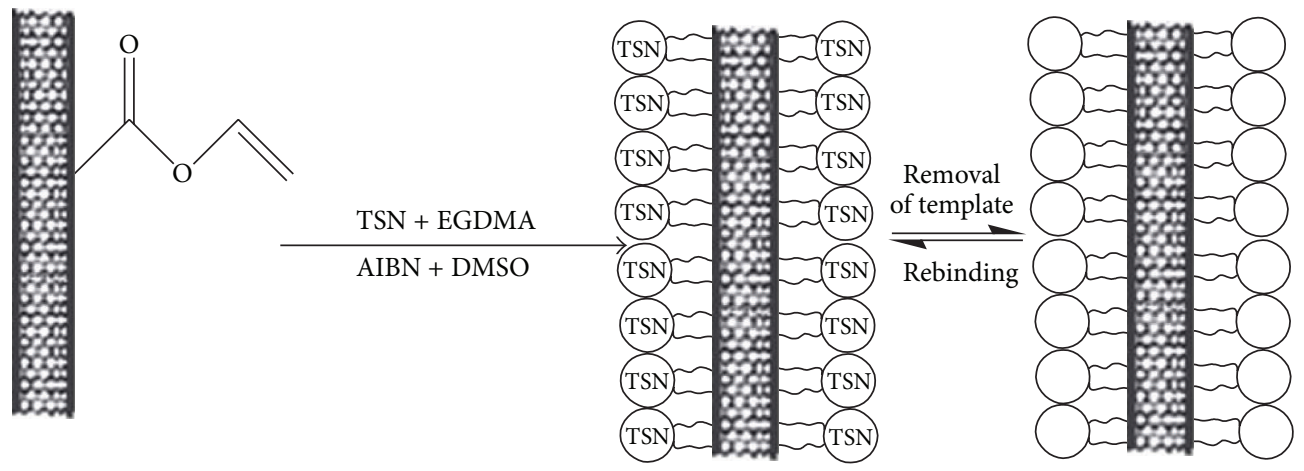

SCHEME 2: Schematic representation for the synthesis of MWCNT-MIP.

Binding conditions were optimised by varying the concentration of TSN solution, solvent, and time. Selectivity of the imprinted membranes was investigated towards cholesterol and progesterone in addition to testosterone.

2.1.7. Column Preparation. A total of $150 \mathrm{mg}$ of the sorbent (molecular imprinted polymer) was poured into the SPE column. A PTFE frit was placed at both ends to prevent loss of the sorbents during the sample loading. Sample solution was delivered into the column by a programmable syringe pump (New Era Pump System). Before loading the sample, SPE cartridges were conditioned by passing $1 \mathrm{~mL}$ methanol and $1 \mathrm{~mL}$ deionised water. Then, $1 \mathrm{~mL}$ of urine sample or progesterone standard solution in deionised water was passed through the column at flow rate of $0.15 \mathrm{~mL} / \mathrm{min}$. The column was washed with $1 \mathrm{~mL}$ water/methanol $(95: 5, \mathrm{v} / \mathrm{v})$ and then eluted with methanol/dichloromethane $(80: 20, \mathrm{v} / \mathrm{v})$ at flow rate of $0.15 \mathrm{~mL} / \mathrm{min}$. The final extract was placed in a water bath $\left(40^{\circ} \mathrm{C}\right)$ and evaporated to dryness under a stream of nitrogen. The residue was dissolved in $100 \mathrm{~mL}$ of methanol.

2.1.8. Urine Samples. Urine of a 2.5-year-old girl was chosen as the blank throughout this study. Urine samples of a girl child were collected and stored at $20^{\circ} \mathrm{C}$ in a freezer until analysis. The spiked urine samples were prepared by adding appropriate amount of testosterone standards in the blank urine. Urine samples were filtered through a $0.2 \mathrm{~mm}$ syringe filter before analysis.

\subsection{Results and Discussion}

2.2.1. Synthesis of MWCNT Based Progesterone Imprinted Polymer. Development of MIP onto the vinyl group functionalized MWCNTs surface is depicted in Scheme 2. Accordingly, the monomer, template, and the cross-linker were mixed together in a test tube followed by the addition of MWCNTs- $\mathrm{CH}=\mathrm{CH}_{2}$ (Table 1). To this prepolymerization mixture, AIBN was added and the polymerization was done. Nonimprinted polymer (NIP) was prepared following the above procedure but in the absence of template. In order to
TABLE 1: Various components for the preparation of MWNTs-MIPs.

\begin{tabular}{lcccc}
\hline Polymer & $\begin{array}{c}\text { MWCNT-CH=CH} \\
(\mathrm{g})\end{array}$ & $\begin{array}{c}\text { EGDMA } \\
(\mathrm{mmol})\end{array}$ & $\begin{array}{c}\text { AIBN } \\
(\mathrm{mg})\end{array}$ & $\begin{array}{c}\text { TSN } \\
(\mathrm{mmol})\end{array}$ \\
\hline MIP & 0.02 & 1.25 & 10 & 0.05 \\
NIP & 0.02 & 1.25 & 10 & 0 \\
\hline
\end{tabular}

reveal the specialty of MWCNTs, an imprinted and nonimprinted polymer was also prepared without carbon nanotube by following the same procedure as described earlier.

\subsubsection{Characterisation}

(i) Fourier Transformation Infrared Spectroscopy (FT-IR). Upon functionalization of MWCNT to MWCNT-COOH we get the peaks corresponding to $3560 \mathrm{~cm}^{-1}(\nu-\mathrm{COOH})$, $2920 \mathrm{~cm}^{-1}(-\mathrm{CH})$, and $1710 \mathrm{~cm}^{-1}(-\mathrm{C}=\mathrm{O})$. On acylation and vinylation, the peaks we got were that of the similar functional groups: for (MWCNT-COCl), $2923 \mathrm{~cm}^{-1}$ ($\mathrm{CH}), 1780 \mathrm{~cm}^{-1}(-\mathrm{C}=\mathrm{O})$, and $850 \mathrm{~cm}^{-1}(-\mathrm{C}-\mathrm{Cl})$ and for MWCNT-CH=CH2 $1740 \mathrm{~cm}^{-1}(\mathrm{C}=\mathrm{O})$, and $1624 \mathrm{~cm}^{-1}(\mathrm{C}-$ C). After the polymerization process to MWCNT-MIP a carbonyl stretching at $1681 \mathrm{~cm}^{-1}$ characteristics of the amide compounds and in addition, acrylamide co-polymer shows typical broad NH stretching at 3368 and $3469 \mathrm{~cm}^{-1}$ were also present.

(ii) Scanning Electron Microscopy (SEM). SEM images of the testosterone imprinted and nonimprinted polymers are shown in Figure 1. In MWCNT-MIP we can clearly understand the nanostructure of the carbon nanotube. In MIP image the porous structure of the polymer is seen.

(iii) X-Ray Diffractogram (XRD). From Figure 2 we can understand that MWCNT having three peaks corresponds to (002), (100), and (004) plane. When we incorporate the MWCNT with conventional polymer two peaks remain corresponding to the MWCNT part. In MIP broad peaks were obtained which indicate the polymer part itself. 


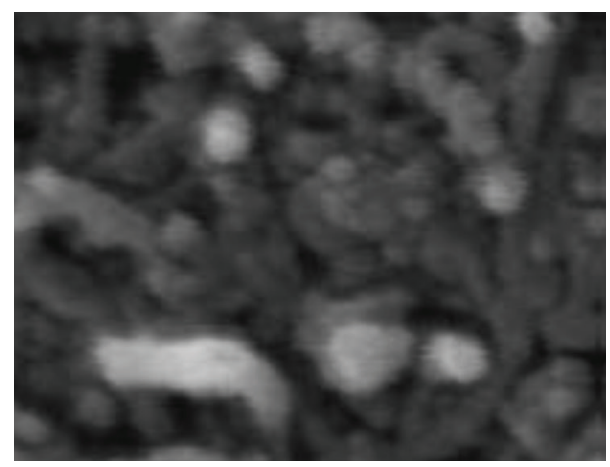

(a)

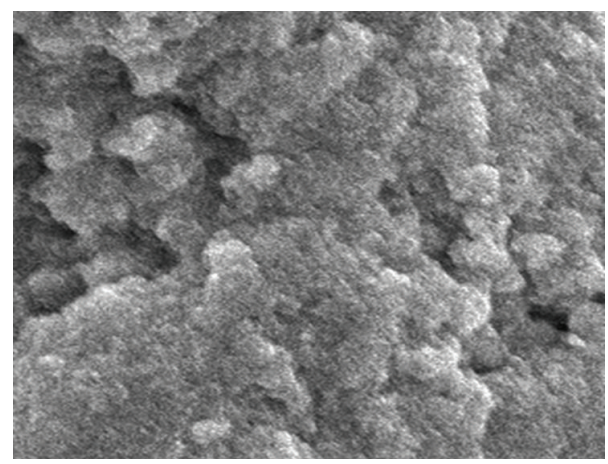

(b)

FIgURE 1: Surface micrographs of TSN (a) MWCNT-MIP and (b) MIP.



FIGURE 2: XRD patterns of TSN (a) MWCNT, (b) MWCNT-MIP and (c) MIP.

\subsubsection{Binding Studies}

\section{Optimisation of the Conditions of PGN Binding}

(i) Effect of Concentration. To evaluate the effect of concentration of TSN on its binding, testosterone solution of different

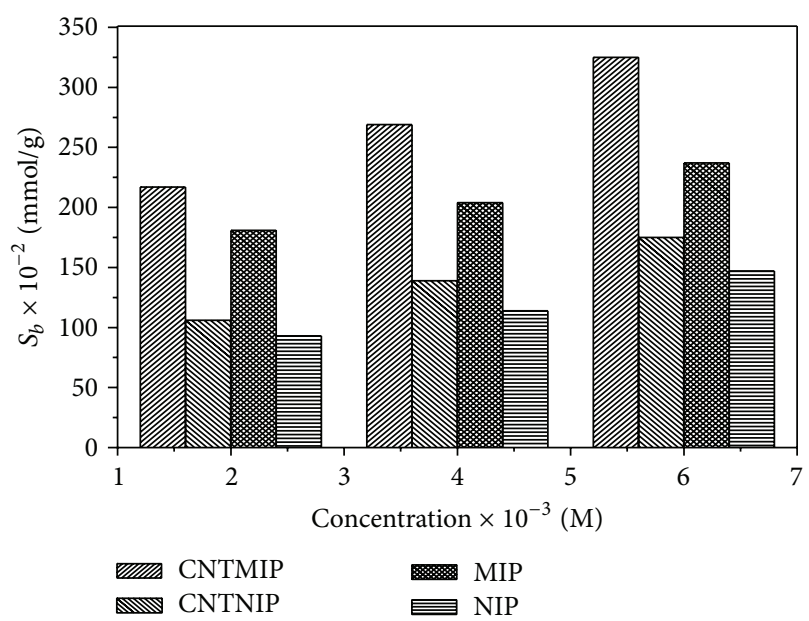

FIGURE 3: Effect of concentration of TSN on the polymers.

concentrations ranging from 1 to $7 \times 10^{-3} \mathrm{M}$ was equilibrated with MWCNT imprinted and nonimprinted polymers and concentration of TSN was followed spectrometrically at $\lambda_{\max }$ $262 \mathrm{~nm}$.

From Figure 3 we can infer that $6 \times 10^{-3} \mathrm{M}$ is the concentration for optimum binding of TSN. In particular nonimprinted polymers also showed a similar retention, which is nonspecific due to the interaction of the testosterone with the nitrile groups of the base polymer and the functional group of the monomer. MWCNT-MIP exhibited much higher retention. The different sorption between each MIP and the corresponding NIP represents the specific binding capacity.

In addition, also the specific binding capacity of the MWCNT-MIP resulted the highest. This behaviour can be ascribed to the acid-base interaction between the weakly acidic phenolic hydrogen of testosterone and the amide group of acrylamide, the nature of which is rather basic. The specific binding results from the formation of recognition sites complimentary to the testosterone in the shape and positioning of functional groups during the imprinting process.

(ii) Effect of Solvent. The effect of solvent on TSN binding was studied using methanol, DMSO, and acetonitrile.

From Figure 4 it is clear that the polymer prepared with methanol is the best for the binding of TSN. Specific binding of TSN was also high for the methanol imprinted polymer. This is mainly due to the porous nature of the polymer.

(iii) Effect of Time. Imprinted polymer reached the saturation limit after $60 \mathrm{~min}$ (Figure 5). The binding specificity behaviour can be attributed to the effective specific recognition properties of the MWCNT-MIP. In the beginning the amount of testosterone bound by the MWCNT-MIP was much higher than the NIP. This difference in time increased until template molecule saturates the complimentary saturation points. Also the PGN took more time to penetrate to the prearranged imprinted sites. But in the control polymer, there are no such prearrangements. 




FIGURE 4: Solvent effect on the binding of MWCNT-MIP and NIP.

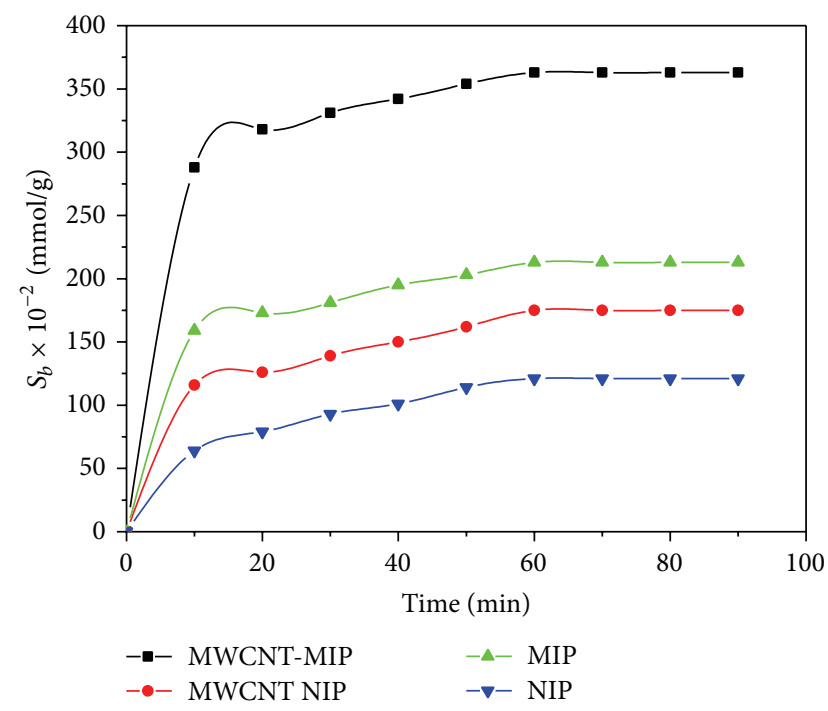

FIGURE 5: The adsorption kinetics process on MWCNT-MIPs/NIPs.

(iv) Desorption and Regeneration Studies. To test the repeat use of the imprinted polymer and to recover the template, the recovery and regeneration tests were conducted. The imprinting process is to be considered as an economical process; it is necessary that the spent imprinted polymers are regenerated. This experiment investigates the extent of TSN desorption from the polymers. Desorption and regeneration studies were carried out using methanol-acetic acid solution.

MWCNT-MIP having a binding quantity of $95.3 \%$ of PGN, 90.8\% was desorbed in methanol-acetic acid solution. The recovery of TSN was decreased from $90.8 \%$ in the first cycle to $87.3 \%$ in the third cycle (Figure 6). The result shows that we can regenerate the spent membranes using methanol-acetic acid solution. The regenerated polymers showed uptake efficiency comparable to that of the fresh one over three cycles.

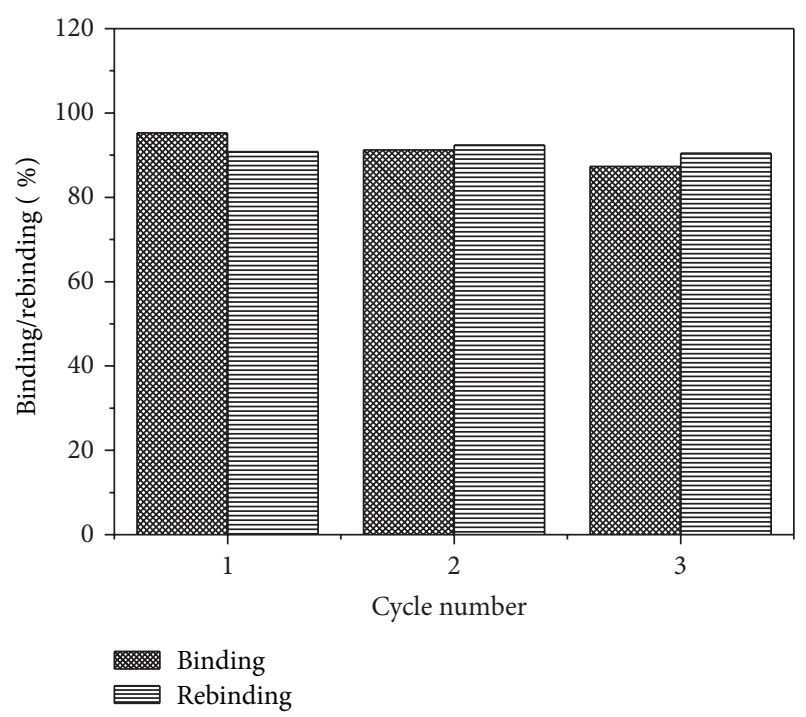

FIGURE 6: Three cycles of TSN binding-rebinding \%.

TABLE 2: Recovery (\%) of PGN after extraction of spiked artificial urine by applying the optimised MISPE protocol.

\begin{tabular}{lccc}
\hline Samples & $\begin{array}{c}\text { Spiked sample } \\
(\mathrm{ng} / \mathrm{mL})\end{array}$ & $\begin{array}{c}\text { Detected sample } \\
(\mathrm{ng} / \mathrm{mL})\end{array}$ & $\begin{array}{c}\text { Recovery } \\
(\%)\end{array}$ \\
\hline 1 & 120 & 115.6 & 96.33 \\
2 & 150 & 147.3 & 98.2 \\
3 & 200 & 197.8 & 98.9 \\
\hline
\end{tabular}

2.2.4. Selectivity. To compare the efficiency of the TSN imprinted polymer, specific binding studies were performed and compared with structurally similar compounds such as progesterone and cholesterol (Figure 7).

From Figure 8 it is clear that the imprinted polymers strongly identify testosterone more than progesterone and cholesterol. This is due to the complementary binding site available in the polymer, which was created in the time of polymerization. This strongly suggests that the imprinted polymer can be effectively used as an absorber for testosterone. Also the MWCNT based polymer system showed higher selectivity to the template TSN.

2.2.5. Analysis of Urine Samples. Urine of a 2.5-year-old boy was spiked with three different amounts of testosterone to reach final concentrations of 120, 150, and $200 \mathrm{ng} / \mathrm{mL}$. Spiked urine samples were processed under the proposed extraction conditions.

In this study, standard addition was used for the determination of testosterone in spiked urine samples. The spectra of blank urine and spiked urine samples are presented in Figure 9. In Table 2, the determined values of testosterone in urine samples are shown. The recoveries were 96-98.9\%, indicating that accuracy of this method is satisfactory for 


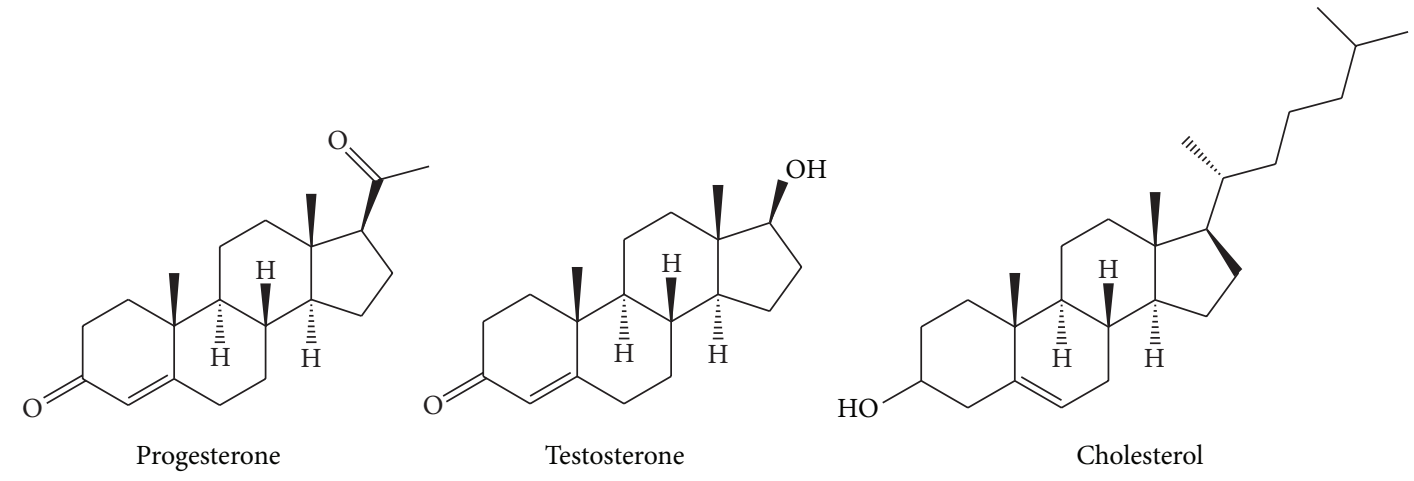

FIGURE 7: Structures of TSN and the competing molecules.

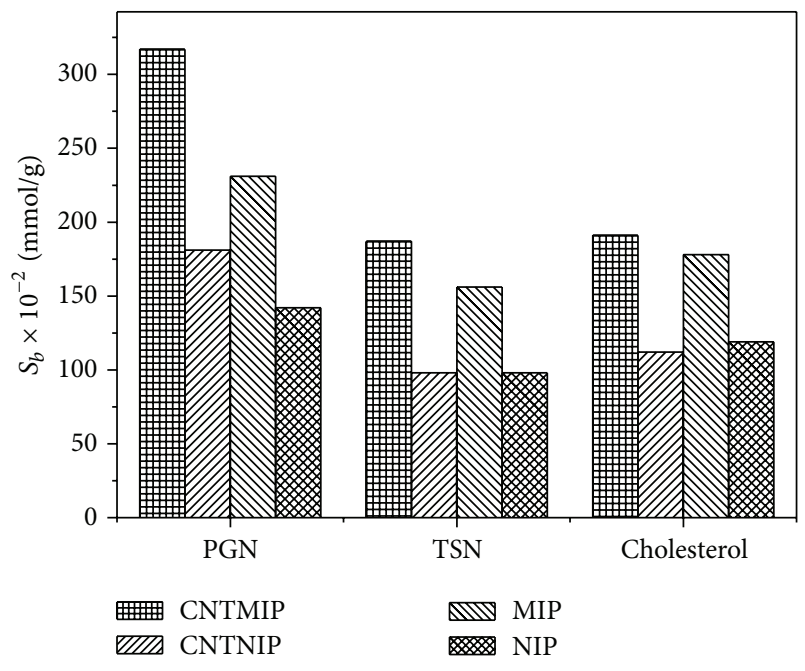

FIGURE 8: Selectivity study with cholesterol and progesterone.

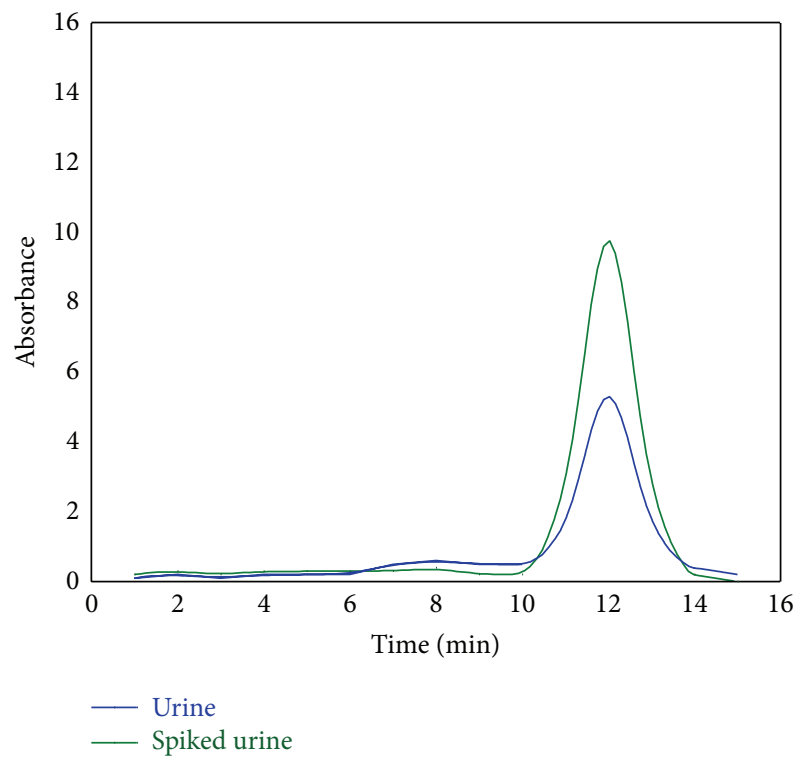

FIGURE 9: Representative chromatogram obtained from extract of the urine and standard mixture of testosterone.

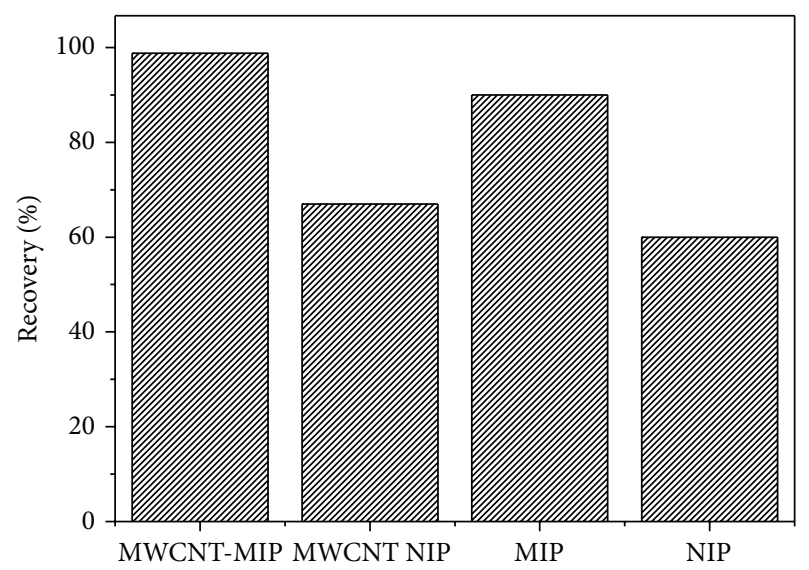

FIGURE 10: The comparison of recovery values between MWCNTMIP and other polymers in elution step.

the analysis of progesterone (Figure 10$)$. The recovery $(R \%)$ was calculated by the following equation:

$$
R=\frac{C_{\text {detected }}}{C_{\text {spiked }}} \times 100 \text {, }
$$

where $C_{\text {detected }}$ and $C_{\text {spiked }}$ are detected and spiked testosterone concentrations, respectively.

\section{Conclusion}

A molecular imprinted polymer impregnated with functionalized MWCNT was prepared. All the imprinted and nonimprinted polymers were characterised by FT-IR, XRD and SEM techniques. Binding studies were conducted. The results emphasised the choice of functionalized MWCNT as the functional monomer appropriate for the imprinting of testosterone and more effective than the conventional polymers. Urine analyses using the prepared polymers were done. The discussed method was highly sensitive and capable of quantifying low circulating levels of testosterone in urine specimens. 


\section{Conflict of Interests}

The authors declare that there is no conflict of interests regarding the publication of this paper.

\section{References}

[1] S.-O. Dima, A. Sarbu, T. Dobre et al., "Molecularly imprinted membranes for selective separations," Materiale Plastice, vol. 46, no. 4, pp. 372-378, 2009.

[2] L. Chen and S. Xuab, "Recent advances in molecular imprinting technology: current status, challenges and highlighted applications," Chemical Society Reviews, vol. 40, pp. 2922-2942, 2011.

[3] G. Wulff and A. Sarhan, "Über die Anwendung von enzymanalog gebauten Polymeren zur Racemattrennung," Angewandte Chemie International Edition in English, vol. 84, p. 364, 1972.

[4] G. Wulff, "Molecular Imprinting in cross-linked materials with the aid of molecular templates-a way towards artificial antibodies," Angewandte Chemie International Edition in English, vol. 34, no. 17, pp. 1812-1832, 1995.

[5] L. Ye and K. Mosbach, "Molecular imprinting: synthetic materials as substitutes for biological antibodies and receptors," Chemistry of Materials, vol. 20, pp. 859-868, 2008.

[6] M. Kempe and K. Mosbach, "Separation of amino acids, peptides and proteins on molecularly imprinted stationary phases," Journal of Chromatography A, vol. 691, no. 1-2, pp. 317-323, 1995.

[7] X. Kan, Y. Zhao, Z. Geng, Z. Wang, and J.-J. Zhu, "Composites of multiwalled carbon nanotubes and molecularly imprinted polymers for dopamine recognition," Journal of Physical Chemistry C, vol. 112, no. 13, pp. 4849-4854, 2008.

[8] S. Iijima, "Helical microtubules of graphitic carbon," Nature, vol. 354, no. 6348, pp. 56-58, 1991.

[9] J. Ričanyová, R. Gadzała-Kopciuch, K. Reiffova, Y. Bazel, and B. Buszewski, "Molecularly imprinted adsorbents for preconcentration and isolation of progesterone and testosterone by solid phase extraction combined with HPLC," Adsorption, vol. 16, no. 4-5, pp. 473-483, 2010.

[10] R. Gadzała-Kopciuch, J. Ričanyová, and B. Buszewski, "Isolation and detection of steroids from human urine by molecularly imprinted solid-phase extraction and liquid chromatography," Journal of Chromatography B, vol. 877, pp. 1177-1184, 2009.

[11] A. Friedel, H. Geyer, M. Kamber et al., "17beta-hydroxy-5alphaandrost-1-en-3-one (1-testosterone) is a potent androgen with anabolic properties," Toxicology Letters, vol. 165, no. 2, pp. 149$155,2006$. 

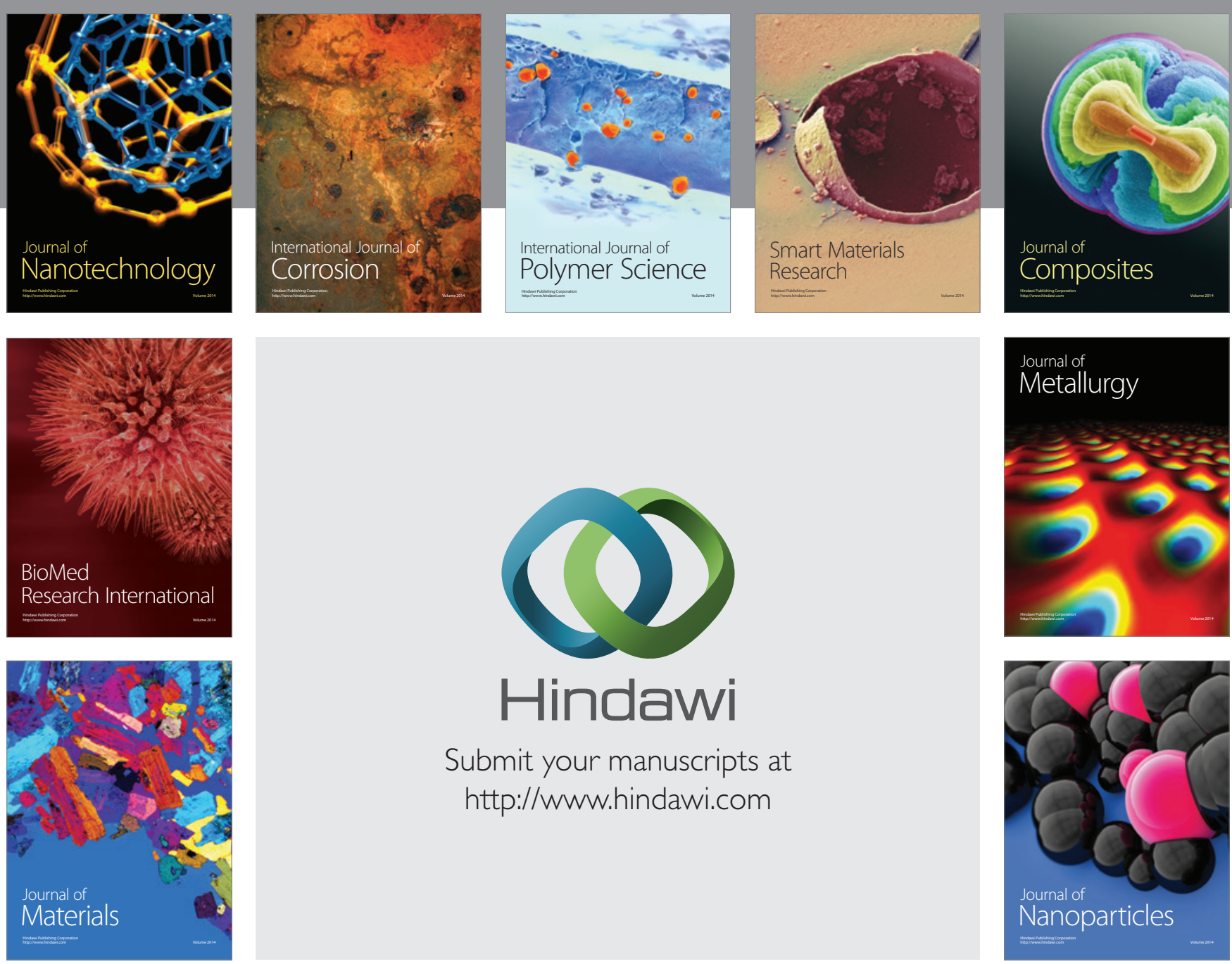

Submit your manuscripts at http://www.hindawi.com
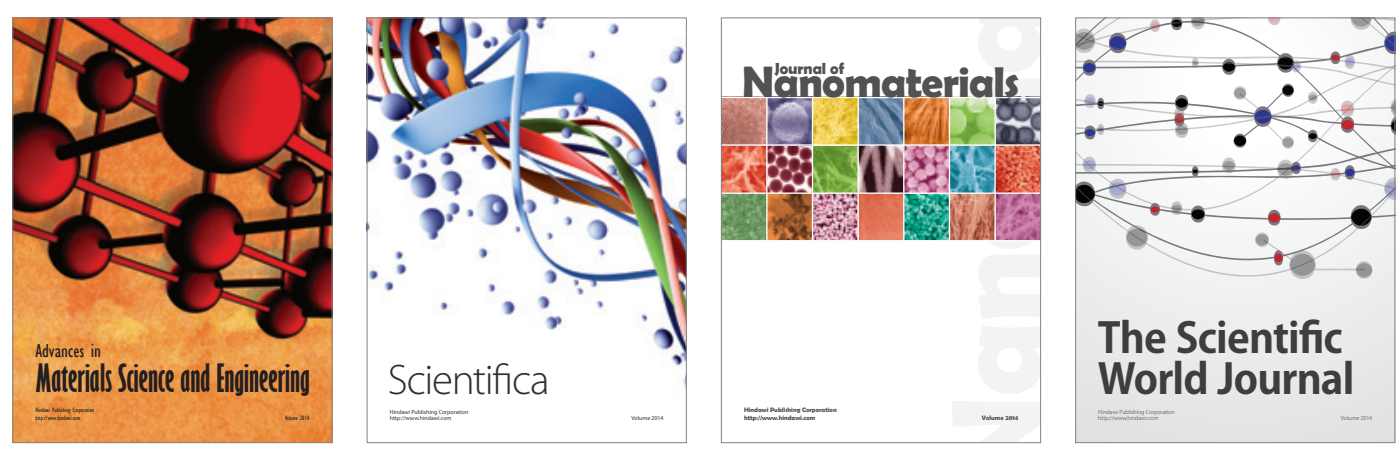

\section{The Scientific World Journal}
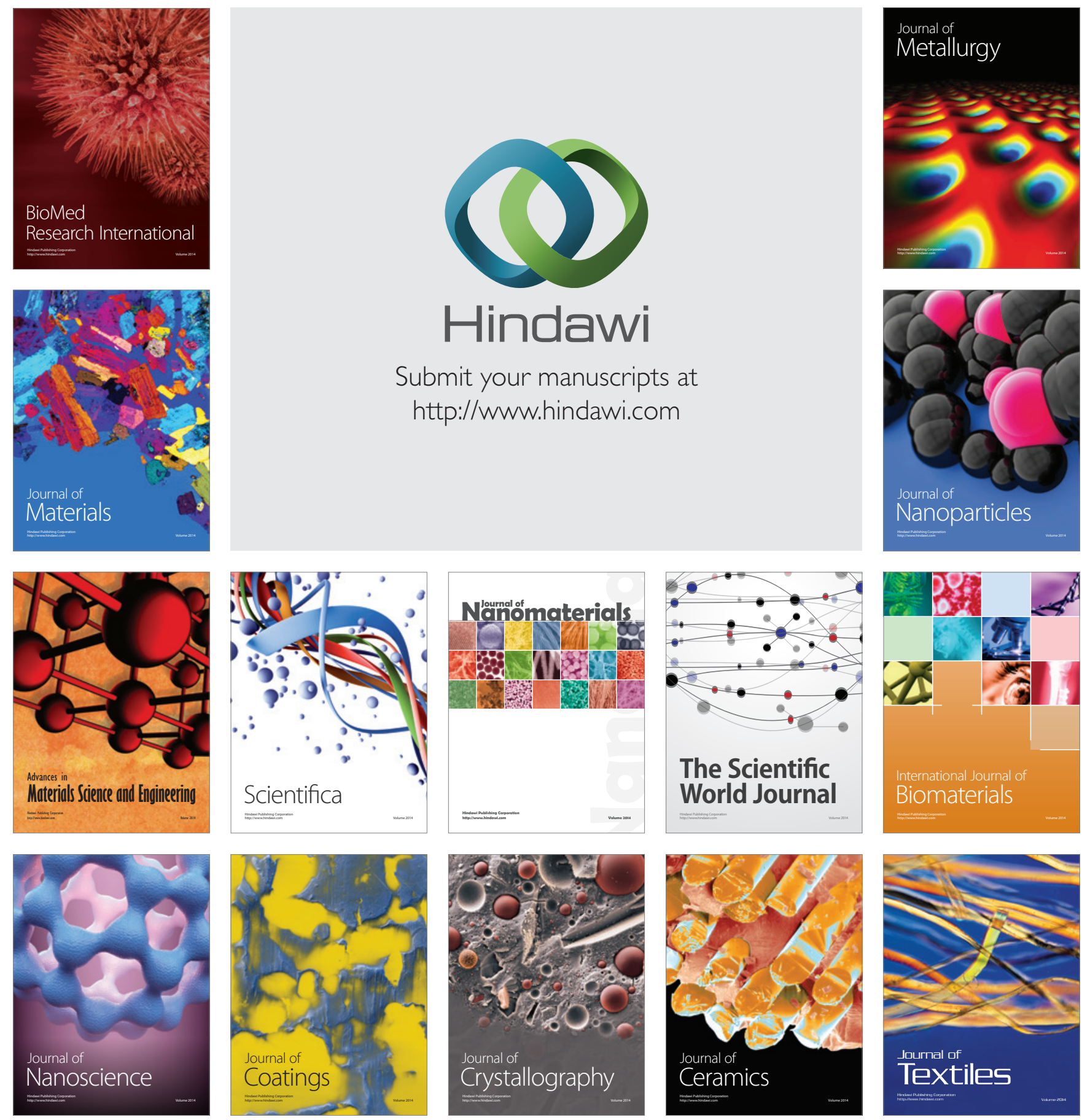